\title{
Reverse time migration using phase crosscorrelation
}

\author{
Jessé C. Costa ${ }^{1}$, Walter E. Medeiros ${ }^{2}$, Martin Schimmel ${ }^{3}$, Flávio L. Santana ${ }^{4}$, and Jörg \\ Schleicher ${ }^{5}$
}

\begin{abstract}
Additional information regarding the continuity and resolution of selected seismic reflectors in reverse time migration (RTM) images can be beneficial for seismic interpretation. We have developed and evaluated new imaging conditions for RTM based on the phase coherence between the forward- and backwardpropagated wavefields. These imaging conditions make use of the instantaneous phase and envelope of the analytical signals of the source and receiver wavefields, in addition to their real parts. Once the analytical wavefields are available, these imaging conditions can be calculated simultaneously with conventional conditions at little or no extra cost. The availability of these fields at each image point enables several alternative ways to define
\end{abstract}

imaging conditions. We explore, in addition to pure phase crosscorrelation (PC), two approaches of amplitude-weighted PC. Our numerical experiments, imaging synthetic and field data sets, indicate that these new imaging conditions provide additional images that can highlight some weak reflectors by locally improving the resolution of RTM images. In our examples, this happens particularly in the deep portions of the seismic images. In addition, reflection events produced at discontinuities are enhanced as sharp signals, suggesting that the proposed imaging conditions can help to delineate stratigraphic and structural features that are harder to see in conventional images. These properties of the PC imaging conditions make them an interesting tool to provide additional information that can aid seismic interpretation in complex structural settings.

\section{INTRODUCTION}

Reverse time migration (RTM) has become the method of choice for seismic imaging in complex geologic settings (Zhang and Sun, 2009). The capability of imaging without dip limitations and the absence of shadow zones have pushed its development. In addition, with a proper change in the boundary conditions for the forwardpropagated wavefield, it is possible even to obtain true amplitude angle-domain common-image gathers (Zhang and Sun, 2009; Yan and Dickens, 2016). RTM images are currently viewed as the most faithful pictures of the earth's subsurface, and there is an increasing demand from interpreters for images showing more stratigraphic details (Etgen et al., 2009). Recent advances in RTM technology have focused on improving efficiency and image quality. The reduction of backscattering noise in RTM has been achieved with postimaging filtering or through modified imaging conditions (Shen and Albertin, 2015; Wang et al., 2016; Du et al., 2017; Moradpouri et al., 2017). Other new imaging conditions can produce amplitude compensations or obliquity corrections, separate events based on their local space-time slope, and reduce backscattered noises (e.g., Guitton et al., 2007; Sava, 2007; Zhang et al., 2007; Chattopadhyay and McMechan, 2008; Schleicher et al., 2008; Costa et al., 2009).

Amplitude-correct images have gained much importance in interpretation. However, sometimes additional, purely stratigraphic images, independent of seismic amplitude information, can be helpful, too. They can allow us to follow reflections, particularly in the deep

Manuscript received by the Editor 5 December 2017; revised manuscript received 1 March 2018; published ahead of production 19 March 2018; published online 6 June 2018.

${ }^{1}$ Universidade Federal do Pará - UFPA, Faculdade de Geofísica, Belém/PA, Brazil and INCT-GP/CNPq — Instituto Nacional de Ciências e Tecnologia em Geofísica do Petróleo, Salvador, Brazil. E-mail: jesse.ufpa@gmail.com.

${ }^{2}$ Universidade Federal do Rio G. do Norte - UFRN, Depto. de Geofísica, Natal/RN, Brazil and INCT-GP/CNPq — Instituto Nacional de Ciências e Tecnologia em Geofísica do Petróleo, Salvador, Brazil. E-mail: walter.ufrn@gmail.com.

${ }^{3}$ Instituto de Ciencias de la Tierra Jaume Almera - CSIC, Barcelona, Spain. E-mail: schimmel@ictja.csic.es.

${ }^{4}$ Universidade Federal do Rio G. do Norte - UFRN, Visiting Researcher - PRH-PB 229, Natal/RN, Brazil. E-mail: flavio@geofisica.ufrn.br.

${ }^{5}$ University of Campinas - Unicamp, DMA/IMECC, Campinas/SP, Brazil and INCT-GP/CNPq — Instituto Nacional de Ciências e Tecnologia em Geofísica do Petróleo, Salvador, Brazil. E-mail: js@ime.unicamp.br.

(C) 2018 Society of Exploration Geophysicists. All rights reserved. 
portions of the seismic images, where amplitude information may become unreliable. In this work, we propose several variations of a new, purely phase-based imaging condition that aims at enhancing stratigraphic and structural features in RTM seismic images. The new imaging conditions require the propagation of the analytical signal of the source and receiver wavefields instead of the usual real wavefields. As a consequence, we have, at each image point, the amplitude and phase fields of the source and receiver wavefields at our disposal. This allows for the use of phase crosscorrelation (PC) (Schimmel, 1999) to complement the classical crosscorrelation (CC) through new mixed imaging conditions. It is important to note that the availability of the analytical wavefields can be used to decompose them into upward- and downward-propagating components (Shen and Albertin, 2015; Wang et al., 2016). In this case, the PC-based imaging conditions can be calculated in addition to conventional imaging conditions at almost no additional cost. The propagation of analytical instead of real wavefields is, at most, twice as expensive.

PC measures the coherence of instantaneous phases (e.g., Bracewell, 1965), and it is explicitly amplitude unbiased. It is determined by the number of phase-coherent time samples. Therefore, it has the potential to enhance weak locally coherent events that would be hard to recognize in a $\mathrm{CC}$ image; this is the motivation to use $\mathrm{PC}$ in an RTM imaging condition. Our numerical experiments, imaging synthetic and field data sets, show that the new imaging conditions using PC indeed improve the resolution of weak-amplitude events in RTM images. In addition, reflection events produced at discontinuities are enhanced as sharp signals, suggesting that the proposed imaging conditions help to better delineate stratigraphic and structural features.

\section{METHODOLOGY}

\section{Phase crosscorrelation}

PC is based on analytic-signal theory. Therein, a complex trace $S(t)=s(t)+i H[s(t)]$ is constructed from the real time series $s(t)$ and its Hilbert transform

$$
H[s(t)]=\frac{1}{\pi} \int_{-\infty}^{\infty} \frac{s(\tau)}{\tau-t} d \tau
$$

The Hilbert transform functions as a $\pi / 2$-phase rotator to compute the orthonormal time series of $s(t)$. The analytic signal is a unique time-dependent representation of the real-valued recorded seismic trace $s(t)$ in the complex domain. It is often expressed by means of an envelope function $A_{S}(t)$ and an instantaneous phase $\varphi_{S}(t)$; i.e., $S(t)=A_{S}(t) \exp \left[i \varphi_{S}(t)\right]$. The instantaneous phase characterizes the waveform complexities as a function of time and has been used to design the phase stack (Schimmel and Paulssen, 1997), an amplitude-unbiased phase-coherent measure based on the sum of phasors $\exp \left[i \varphi_{S}(t)\right]$.

Let $S(t)$ and $R(t)$ be two analytical signals associated with two time series $s(t)$ and $r(t)$, respectively, with envelopes $A_{S}(t)$ and $A_{R}(t)$ and instantaneous phases $\varphi_{S}(t)$ and $\varphi_{R}(t)$. In an analogy to CC, PC between $S(t)$ and $R(t)$ at time lag $\tau$ in a time window $\left[t_{0}, t_{0}+W\right]$ is defined as

$$
\operatorname{PC}(\tau, W ; S, R ; \nu)=\frac{1}{W} \int_{t_{0}}^{t_{0}+W} \Psi_{\tau}\left(\varphi_{S}(t), \varphi_{R}(t) ; \nu\right) d t,
$$

where the expression

$$
\begin{gathered}
\Psi_{\tau}\left(\varphi_{S}(t), \varphi_{R}(t) ; \nu\right)=\frac{1}{2^{\nu}}\left[\left|\exp \left\{i \varphi_{S}(t+\tau)\right\}+\exp \left\{i \varphi_{R}(t)\right\}\right|^{\nu}\right. \\
\left.-\left|\exp \left\{i \varphi_{S}(t+\tau)\right\}-\exp \left\{i \varphi_{R}(t)\right\}\right|^{\nu}\right]
\end{gathered}
$$

substitutes the product $\Phi_{\tau}(t)=s(t+\tau) r(t)$ appearing in CC. Above, $W$ is the correlation window length and $\nu$ is a heuristic parameter that permits tuning of the sensitivity of the phase stacks. According to Schimmel and Paulssen (1997), this parameter permits the tuning of the transition between more and less coherent signals; i.e., a higher $\nu$ value leads to stronger attenuation of less correlated signals and, thus, helps to better highlight phase-coherent signals. For instance, Tanaka (2005) uses the phase stack with $\nu=100$ to attenuate side lobes in array responses to enable a less ambiguous detection of weak amplitude signals from the core of the earth.

Phase correlation works as follows. When two time series present a high waveform similarity with the same polarity around lag $\tau$, their phasors are approximately equal, $\varphi_{R}(t) \approx \varphi_{S}(t+\tau)$, so that expression 3 reduces to

$$
\Psi_{\tau}\left(\varphi_{S}(t), \varphi_{R}(t) ; \nu\right) \approx \frac{1}{2^{\nu}}\left[\left|2 \exp \left\{i \varphi_{R}(t)\right\}\right|^{\nu}-0\right]=1
$$

whereas for reverse polarity, $\varphi_{R}(t) \approx-\varphi_{S}(t+\tau)$, resulting in

$$
\Psi_{\tau}\left(\varphi_{S}(t), \varphi_{R}(t) ; \nu\right) \approx \frac{1}{2^{\nu}}\left[0-\left|2 \exp \left\{i \varphi_{R}(t)\right\}\right|^{\nu}\right]=-1
$$

On the other hand, for two time series that do not present a waveform similarity around lag $\tau$, PC (equation 2) represents a sum over the sine and cosine functions, whose integral approximately vanishes if the window is sufficiently large. In summary, PC is a real-valued functional that measures anticorrelated and correlated waveform similarity between -1 and 1 , in full analogy to a normalized version of CC. Note that because PC is actually a correlation of phasors, there are no problems with phase wrapping. For further details, please refer to Schimmel and Paulssen (1997) or Schimmel (1999).

PC has been successfully applied in seismic interferometry studies, in which it improves the extraction of small signals (Schimmel et al., 2011) for monitoring or imaging. The monitoring studies benefit from the amplitude-unbiased approach through a fast convergence to a stable medium response and consequently improved time resolution (e.g., D'Hour et al., 2015). In seismic imaging studies, PC has been used mainly for signal extraction, such as coda phases, to map upper mantle discontinuities (Bonatto et al., 2015) and empirical Green's functions for global ambient noise tomography (Haned et al., 2015). Gaudot et al. (2015) take advantage of PCs statistical properties to detect and image a noise source. To our knowledge, PC has not been used before to build RTM imaging conditions.

According to Schimmel (1999), PC shows higher sensitivity to coherence than $\mathrm{CC}$, regardless of the event amplitude. This observation is in good qualitative agreement with the known fact that instantaneous phase, when used as a seismic attribute, shows finer layering details and improved reflection continuity (Barnes, 2007). 


\section{PC imaging conditions}

Let $p_{S}(\mathbf{x}, t)$ and $p_{R}(\mathbf{x}, t)$ denote the forward-propagated source wavefield and the backward-propagated receiver wavefield, respectively. Then, any imaging condition in RTM relies on the assumption that, for each shot, events in $p_{S}(\mathbf{x}, t)$ and $p_{R}(\mathbf{x}, t)$ should coincide in space and time at every scattering point in the subsurface (Claerbout, 1971). Given a migration velocity field consistent with the data, this condition can focus reflections and diffracted events at their correct position. The classical implementation of this assumption is a crosscorrelation; i.e.,

$$
I_{\mathrm{CC}}(\mathbf{x})=\sum_{S} \int_{0}^{T} p_{S}(\mathbf{x}, t) p_{R}(\mathbf{x}, t) d t
$$

where $I_{\mathrm{CC}}(\mathbf{x})$ represents the seismic image at a subsurface point $\mathbf{x}$, calculated using Claerbout's classical RTM imaging condition, and where $T$ is the maximum recorded time. This original imaging condition has been revised by several authors aiming to improve the quality of RTM images (Yoon and Marfurt, 2006; Guitton et al., 2007; Zhang et al., 2007; Schleicher et al., 2008; Costa et al., 2009). Their contributions provide amplitude correction, reduction of the backscattered noise, better illumination compensation, and obliquity factor corrections. For comparison, we also use in this work the version of Guitton et al. (2007),

$$
I_{\mathrm{CC}}^{i c}(\mathbf{x})=\frac{\sum_{S} \int_{0}^{T} p_{S}(\mathbf{x}, t) p_{R}(\mathbf{x}, t) d t}{\left\langle\sum_{S} \int_{0}^{T} p_{S}(\mathbf{x}, t) p_{S}(\mathbf{x}, t) d t\right\rangle},
$$

which provides an amplitude correction by illumination compensation. Here, $\langle\cdot\rangle$ represents a local averaging operation in a small window around position $\mathbf{x}$. This averaging was proposed by Guitton et al. (2007) to stabilize the division, because some points $\mathbf{x}$ in the image may be poorly illuminated.

If a different look at the subsurface properties is desirable, $\mathrm{PC}$ can be used to focus RTM images with enhanced seismic-phase information and without seismic-amplitude dependence. In that case, it is necessary to propagate the analytical signals $P_{S}(\mathbf{x}, t)$ and $P_{R}(\mathbf{x}, t)$ associated with the source wavefield $p_{S}(\mathbf{x}, t)$ and the receiver wavefield $p_{R}(\mathbf{x}, t)$ into the subsurface. For this purpose, one needs to add the Hilbert transform of the real wavefields as an imaginary part and then proceed with the chosen propagation algorithm applied to the complex wavefields. Then, at every time frame $t$ and at every position $\mathbf{x}$ in the subsurface, the complex fields

$$
P_{R}(\mathbf{x}, t)=A_{R}(\mathbf{x}, t) \exp \left[i \varphi_{R}(\mathbf{x}, t)\right]
$$

and

$$
P_{S}(\mathbf{x}, t)=A_{S}(\mathbf{x}, t) \exp \left[i \varphi_{S}(\mathbf{x}, t)\right]
$$

are available.

With this information, the simplest PC-based imaging condition is to stack over the zero-lag version of PC in equation 2; i.e.,

$$
\begin{aligned}
I_{\mathrm{PC}}(\mathbf{x}) & =\frac{1}{N_{S}} \sum_{S} \mathrm{PC}\left(\tau=0, T ; P_{R}(\mathbf{x}, t), P_{S}(\mathbf{x}, t) ; \nu\right) \\
& =\frac{1}{N_{S} T} \sum_{S} \int_{0}^{T} \Psi_{0}\left(\varphi_{S}(\mathbf{x}, t), \varphi_{R}(\mathbf{x}, t) ; \nu\right) d t,
\end{aligned}
$$

where $N_{S}$ denotes the number of shots.

Imaging condition 10 does not contain any seismic amplitude information at all and will show all coherent events in the seismic image with comparable amplitudes, including all migration artifacts. This may lead to an image that is rather hard to interpret. Therefore, we also tested several possibilities to include the seismic amplitudes as weights in imaging condition 10, so as to favor seismic signals over artifacts. Several alternative imaging conditions can be conceived of, which make use of the two real wavefields $p_{S}(\mathbf{x}, t)$ and $p_{R}(\mathbf{x}, t)$ and the four analytical fields $A_{R}(\mathbf{x}, t)$, $\varphi_{R}(\mathbf{x}, t), A_{S}(\mathbf{x}, t)$, and $\varphi_{S}(\mathbf{x}, t)$. The two most straightforward ideas are the amplitude-weighted PC imaging condition:

$I_{\mathrm{PC}}^{p}(\mathbf{x})=\frac{\sum_{S} \int_{0}^{T}\left|p_{S}(\mathbf{x}, t) p_{R}(\mathbf{x}, t)\right| \Psi_{0}\left(\varphi_{S}(\mathbf{x}, t), \varphi_{R}(\mathbf{x}, t) ; \nu\right) d t}{\sum_{S} \int_{0}^{T}\left|p_{S}(\mathbf{x}, t) p_{R}(\mathbf{x}, t)\right| d t}$

and the envelope-weighted PC imaging condition:

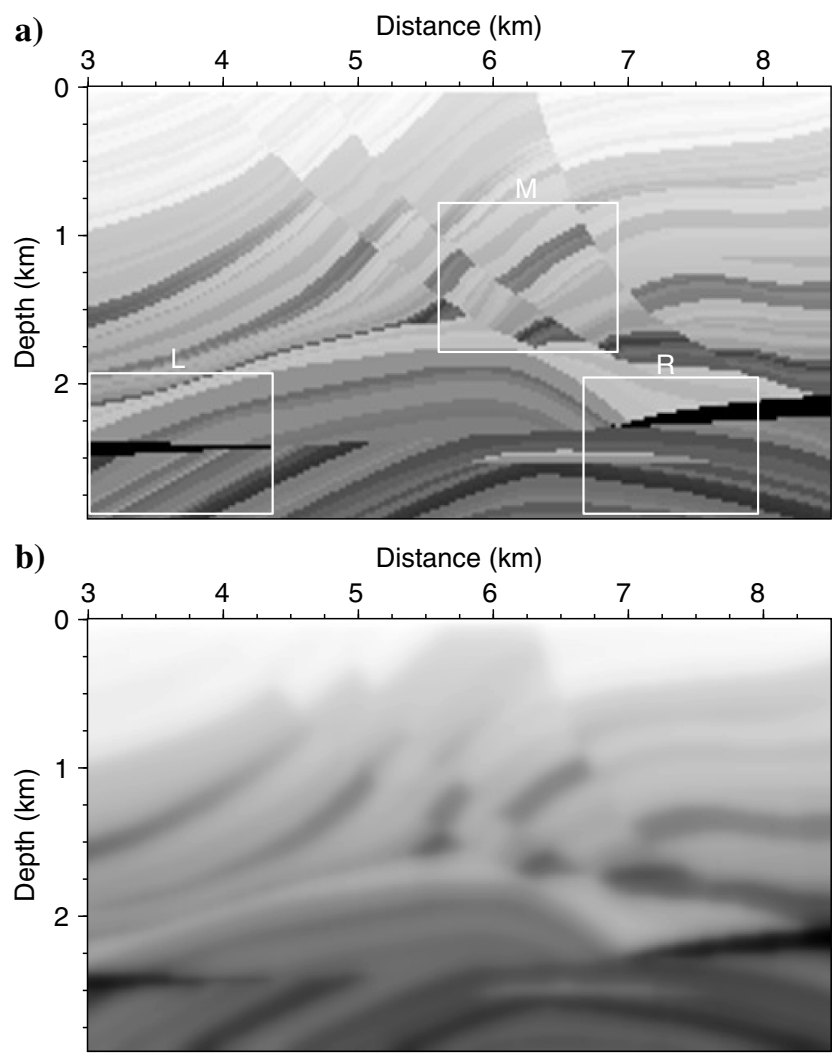

Figure 1. Marmousi velocity model. (a) True model (rectangles indicate magnified areas) and (b) smoothed migration velocity model. 
$I_{\mathrm{PC}}^{A}(\mathbf{x})=\frac{\sum_{S} \int_{0}^{T} A_{S}(\mathbf{x}, t) A_{R}(\mathbf{x}, t) \Psi_{0}\left(\varphi_{S}(\mathbf{x}, t), \varphi_{R}(\mathbf{x}, t) ; \nu\right) d t}{\sum_{S} \int_{0}^{T} A_{S}(\mathbf{x}, t) A_{R}(\mathbf{x}, t) d t}$.

Note that the denominators in equations 11 and 12 normalize the weights in the numerators, so as to keep the image amplitudes at different image points comparable. Because the denominators involve the amplitudes of the source and receiver wavefields, there

a)

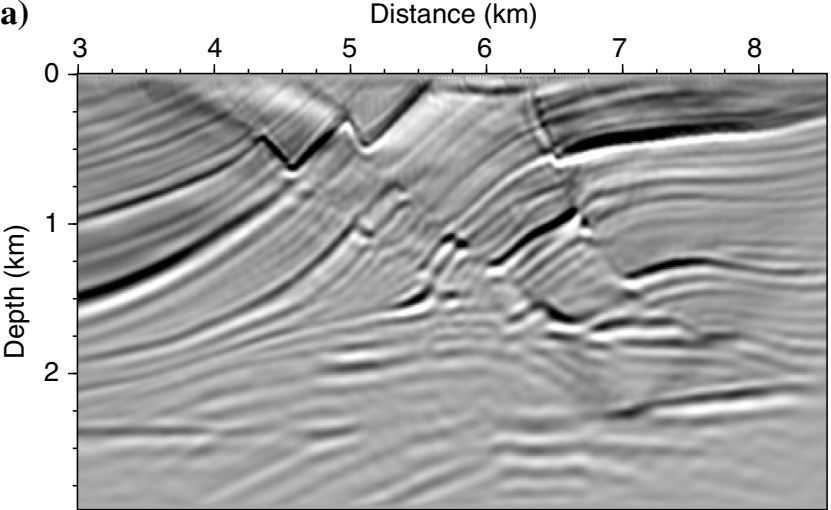

b)

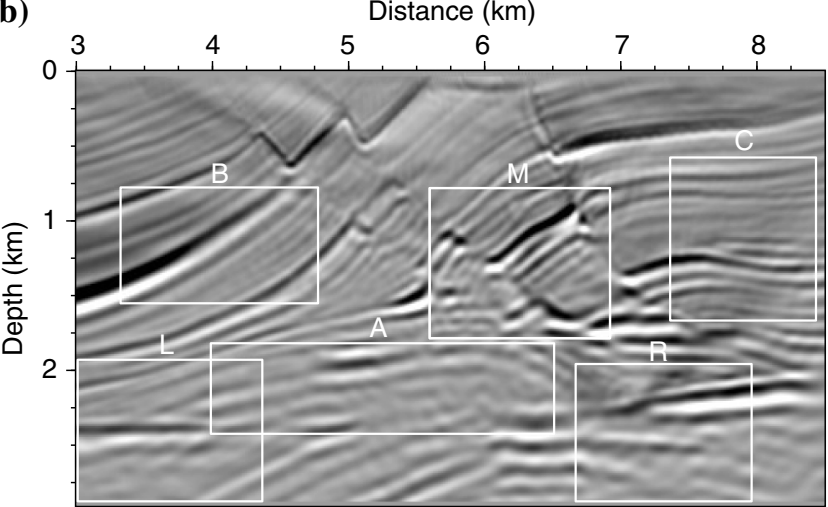

Figure 2. Classic crosscorrelation RTM image (a) without and (b) with illumination compensation (conditions $I_{\mathrm{CC}}(\mathbf{x})$ and $I_{\mathrm{CC}}^{i c}(\mathbf{x})$ in equations 6 and 7, respectively). Rectangles L, M, and R indicate the magnified areas, rectangles $\mathrm{A}, \mathrm{B}$, and $\mathrm{C}$ indicate the comparison areas.

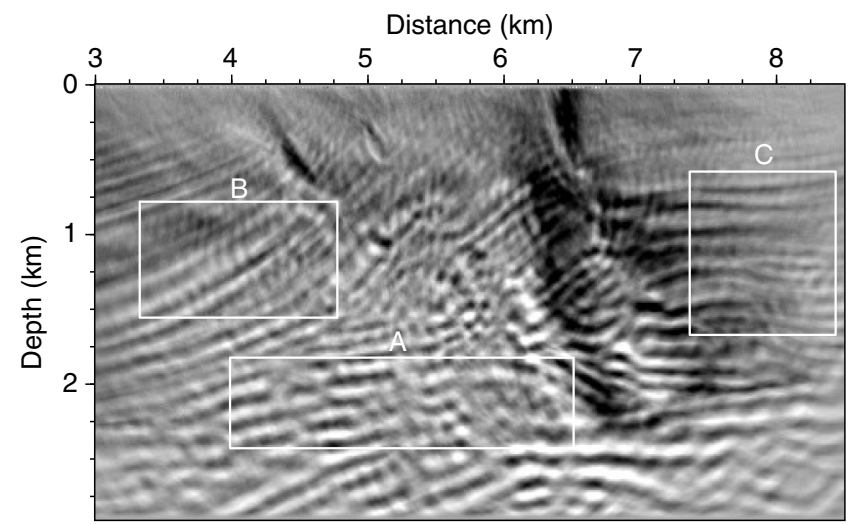

Figure 3. The PC RTM image (condition $I_{\mathrm{PC}}(\mathbf{x})$ in equation 10 ) with $\nu=100$. Compare rectangles A, B, and C with those in Figure $2 \mathrm{~b}$. is practically no risk for them to vanish. Therefore, no need to stabilize the divisions in equations 11 and 12 arose in our numerical examples.

The amplitude- and envelope-weighted PC imaging conditions $I_{\mathrm{PC}}^{p}(\mathbf{x})$ and $I_{\mathrm{PC}}^{A}(\mathbf{x})$ are weighted averages of the zero-lag PC integrand $\Psi_{0}\left(\varphi_{R}, \varphi_{S} ; \nu\right)$, with weights making use of seismic amplitude information. For $I_{\mathrm{PC}}^{p}(\mathbf{x})$, the weights are composed of the moduli of the real wavefields, whereas for $I_{\mathrm{PC}}^{A}(\mathbf{x})$, the weights are determined by the analytical envelope amplitudes. It must be stressed that the weights do not carry any phase information, but they are designed to attenuate coherent low-amplitude migration artifacts. In addition to the unweighted PC imaging condition of equation 10 and these two weighted versions, we tested several other versions, none of which produced results as good as these.

\section{NUMERICAL EXPERIMENTS}

\section{Marmousi data}

To investigate the properties of these new imaging conditions on RTM images, besides the effect of parameter $\nu$, we performed numerical experiments using the Marmousi data set. As a reference for comparison, we use the CC images obtained with equation 6 and its illumination-compensated version, equation 7 .

For our numerical tests in the 2D acoustic approximation, we compute the analytical solution of the acoustic wave equation using

a)

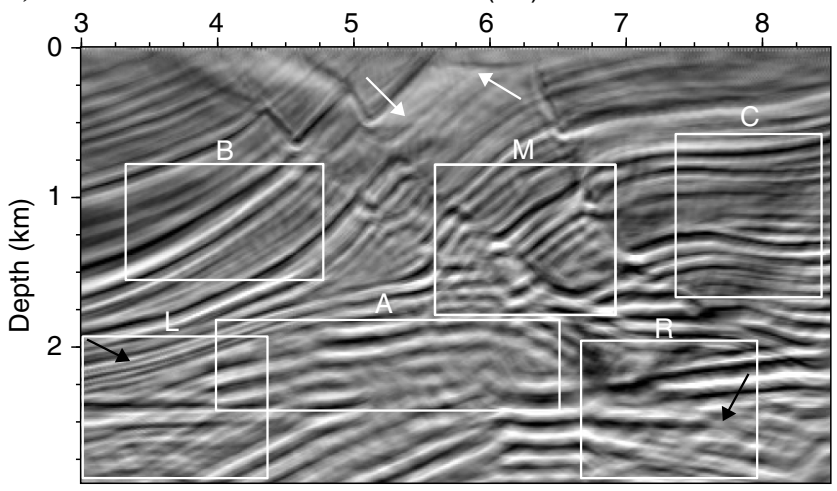

b)

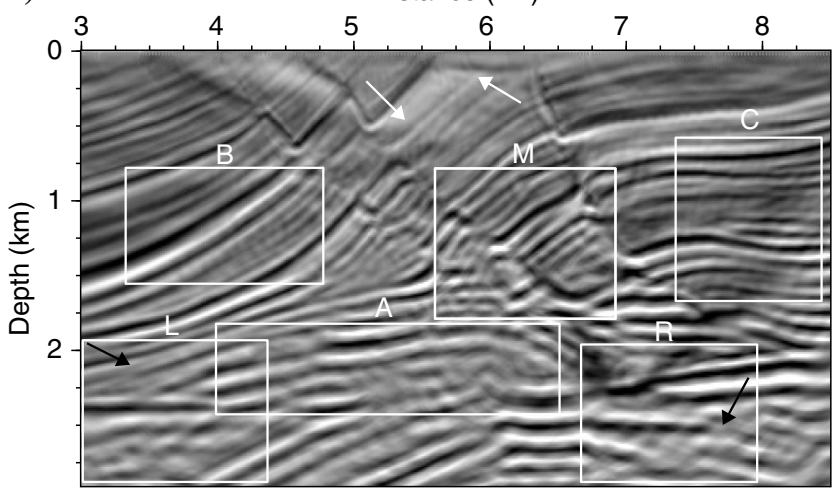

Figure 4. (a) Amplitude-weighted and (b) envelope-weighted PC RTM images (conditions $I_{\mathrm{PC}}^{p}(\mathbf{x})$ and $I_{\mathrm{PC}}^{A}(\mathbf{x})$ in equations 11 and 12 , respectively) with $\nu=100$. Compare rectangles $\mathrm{A}, \mathrm{B}$, and $\mathrm{C}$ with those in Figures $2 b$ and 3. Rectangles L, R, and M indicate the magnified areas. 
a finite-difference scheme of second order in time and 14th order in space. To reduce numerical dispersion and numerical anisotropy, we optimized the spatial operators according to Holberg (1987). The efficiency of the analytical-wavefield propagation can be further improved using the prescriptions of Pestana and Revelo (2017).

The migration velocity model (Figure $1 \mathrm{~b}$ ) is a smoothed version of the Marmousi velocity model (Figure 1a) sampled on a uniform grid with $12 \mathrm{~m}$ interval. The RTM images were computed with a Ricker source wavelet with peak frequency equal to $12 \mathrm{~Hz}$. The averaging operation in equation 7 is computed using a $5 \times 5$ square box around each imaging point.

Figure 2 shows the CC reference images with and without illumination compensation (conditions $I_{\mathrm{CC}}(\mathbf{x})$ in equation 6 , and $I_{\mathrm{CC}}^{i c}(\mathbf{x})$ in equation 7). To allow for a comparison of the pure effect of the imaging conditions, we have refrained from applying any filters that could be used in all images to reduce the low-frequency backscattering noise. Figure 3 shows the image produced using the PC imaging condition (condition $I_{\mathrm{PC}}(\mathbf{x})$ in equation 10) with $\nu=100$. In spite of the somewhat unstable first impression of the latter image, closer inspection reveals some interesting features, particularly in the deeper part of the image. For example, the geometry of the anticlines less than $2 \mathrm{~km}$ depth (see the rectangle labeled $\mathrm{A}$ in Figures $2 \mathrm{~b}$ and 3 ) is more easily recognizable. In addition, some stratigraphic features are enhanced, such as, for instance, the bedding structures around depth $1.25 \mathrm{~km}$ at position $4 \mathrm{~km}$ (rectangle B) and around depth $1.25 \mathrm{~km}$ at position $8 \mathrm{~km}$ (rectangle C).

The rather unstable aspect of the image in Figure 3 is caused by the fact that the PC-based imaging condition of equation 10 is completely amplitude independent. As a result of this amplitude insensitivity, migration artifacts with low amplitudes in the reference images of Figure 2 are amplified. Moreover, the low-frequency backscattering is stronger than in the reference images. Apparently, this noise is perceived as coherent by the phasors in equation 10 and thus also enhanced. Overall, we conclude that although it enhances these kinds of undesirable effects, a PC-based imaging condition can be useful to highlight structures that might be missed in a conventional image.

To take advantage of the high sensitivity of the PC imaging condition while improving the robustness regarding artifacts, we studied the amplitude- and envelope-weighted PC imaging conditions given in equations 11 and 12 . The resulting images using $\nu=100$ are shown in Figure 4 . These images are indeed easier to interpret because most of the undesirable artifacts present in Figure 3 are strongly attenuated. However, the better stratigraphic quality of the structures mentioned above (rectangles $\mathrm{A}, \mathrm{B}$, and $\mathrm{C}$ ) is preserved.

When compared with Figure 2, we may even say that the weighted-PC RTM images present higher resolution. This is corroborated by the 2D Fourier spectra shown in Figure 5, which show extended areas with nonzero contributions in the spectra of the weighted PC images. Figure $5 \mathrm{~d}$ compares the areas covered by these spectra with amplitudes that are higher than half a percent of the peak amplitude. Clearly, the spectra of the weighted PC images retain higher wavenumbers than the $\mathrm{CC}$ image, with the amplitude-weighted PC image covering the largest area. We notice a pronounced effect in the vertical direction, indicating a higher resolution and sharper representation of discontinuities in this direction. The slight slant of all three spectra indicates the prominent overall dip direction in the Marmousi model.

In the weighted PC images of Figure 4, we recognize some are as with improved resolution of subtle stratigraphic and structural features. Figure 6 shows magnifications of three of these areas, where improvements are most prominent, and compares them with the velocity and reflectivity models. The areas shown in the left, middle, and right columns of Figure 6 correspond to the rectangles labeled L, M, and R, respectively, in Figures 1a, 2b, and 4.

The magnifications in the left column of Figure 6 show that the dipping layers, composing the left flank of the anticline less than $2 \mathrm{~km}$ depth (rectangle L in Figures 1a, 2b, and 4), are better imaged in the weighted PC images than in the reference image. In the same magnifications, we also recognize a resolution improvement of the thin-layer sequence in the region below the salt wedge. In spite of the also enhanced horizontal artifacts, probably internal reverberations in the salt wedge, the dipping layer cake is interpretable in the PC images and compares favorably with the reflectivity model, particularly in the amplitude-weighted version. This demonstrates that the PC-based imaging conditions can help to resolve subtle stratigraphic features in the model (see again Figure 1a).
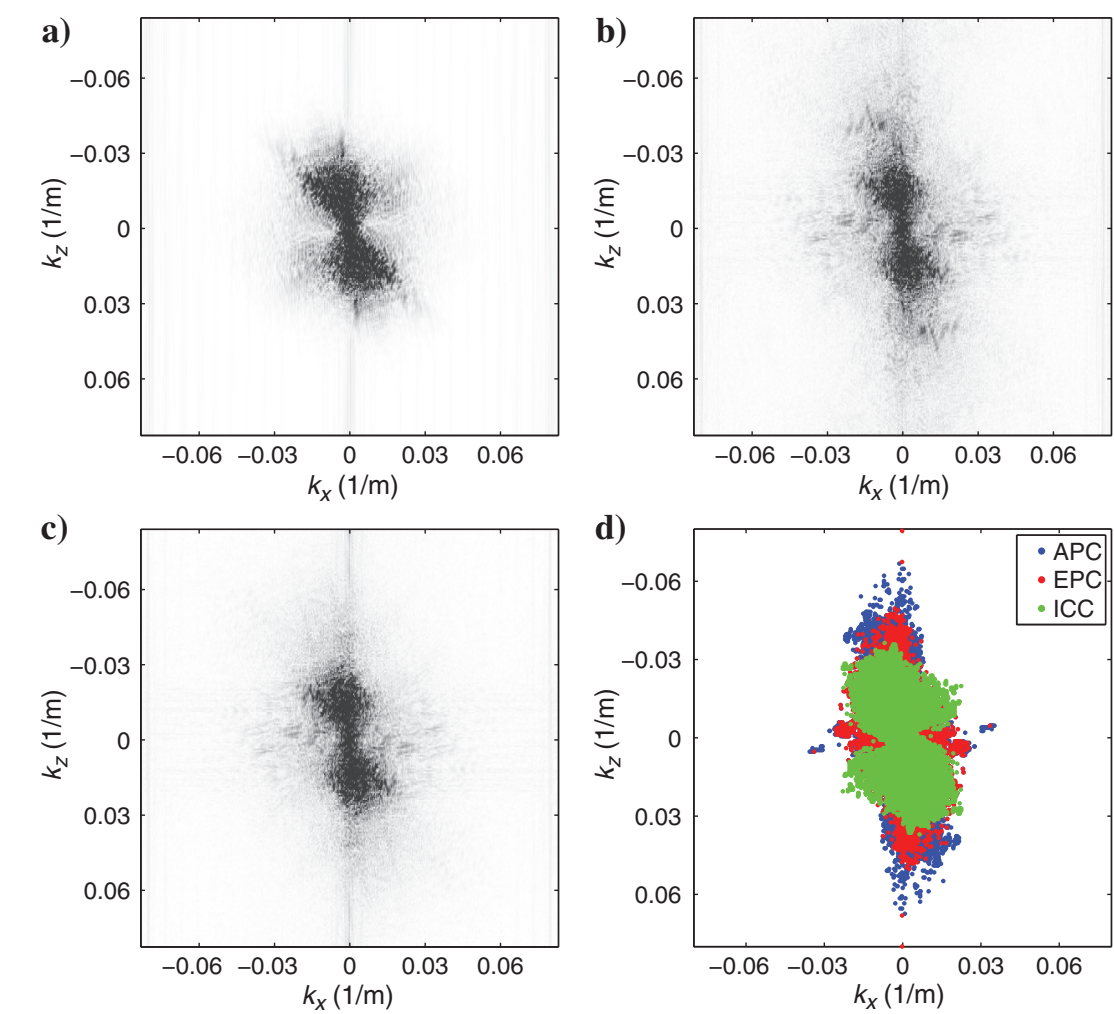

d)

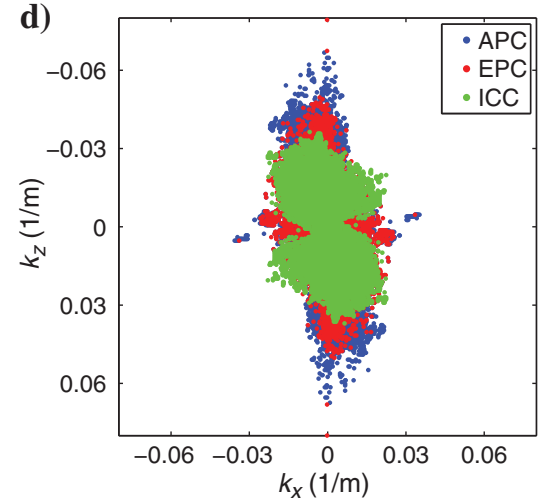

Figure 5. The 2D Fourier spectra of (a) the reference $\mathrm{CC}$ image (with illumination compensation) in Figure 2b, (b) the amplitude-weighted PC image in Figure 4a, and (c) the envelope-weighted PC image in Figure 4b. (d) Superimposed spectra: illumination-compensated CC image (red, ICC), amplitude-weighted PC image (blue, APC), and envelope-weighted PC image (green, EPC). 
Some structural features are also enhanced. For example, the strata truncations against the fault plane, at approximately $1 \mathrm{~km}$ depth and position $6 \mathrm{~km}$ (rectangle M, middle column in Figure 6), are more evident in the weighted-PC RTM images than in the reference image. As a final example of the resolution increase, observe that around the target region at approximately $2.5 \mathrm{~km}$ depth and position $7 \mathrm{~km}$ (rectangle R, right column in Figure 6), the reflectors in the reservoir region at the top of an anticline and below a truncation subhorizontal surface are more easily seen in the weightedPC RTM images than in the reference image.
The differences between the two weighted-PC images (Figure 4) are rather subtle. On the one hand, the envelope-weighted PC imaging condition (Figure $4 \mathrm{~b}$ ) appears to better delineate the fault and the stratigraphic geometry around the fault corners in the depth interval $0-1 \mathrm{~km}$ and position $5-7 \mathrm{~km}$ (white arrows) than its amplitude-weighted counterpart. A possible explanation is that the envelope weight imposes better stability on the PC imaging condition, because the envelopes present smoother variations than the amplitudes. On the other hand, the amplitude-weighted PC imaging condition (Figure 4a) improves the resolution in rectangles $\mathrm{L}$ and $\mathrm{R}$ a)

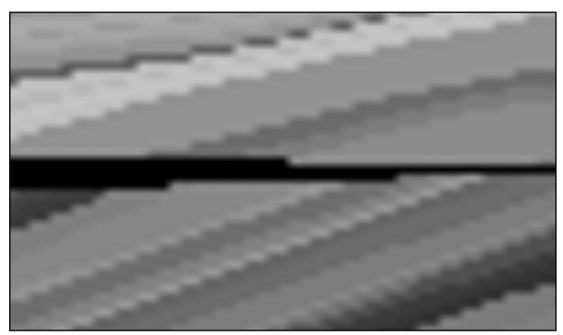

b)

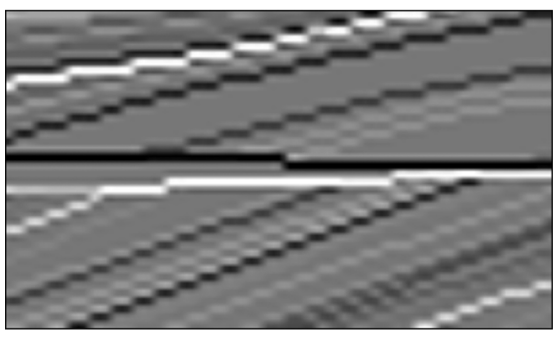

c)

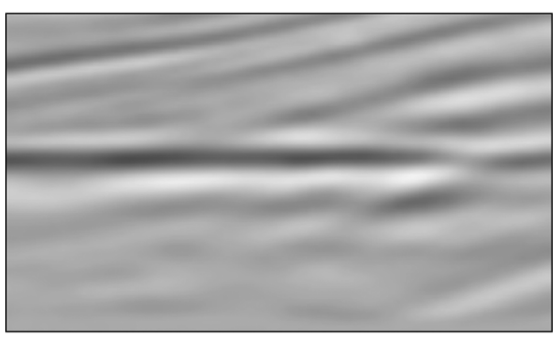

d)

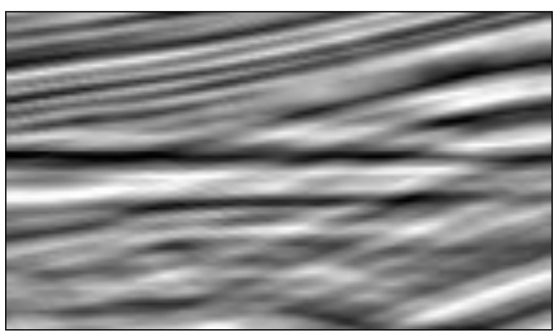

e)

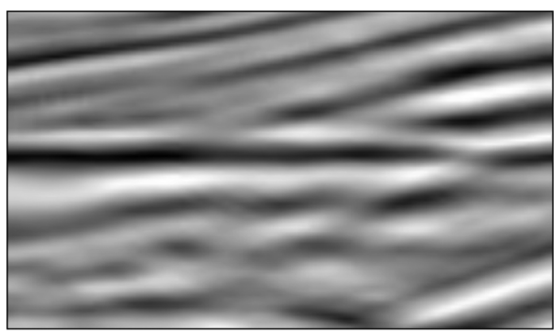

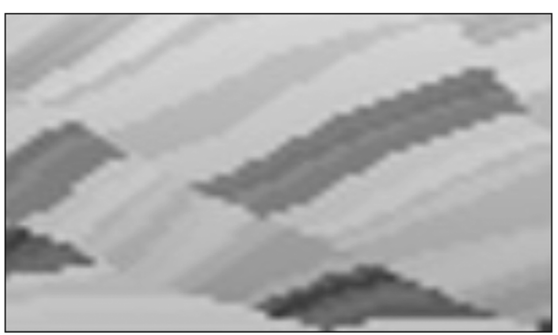
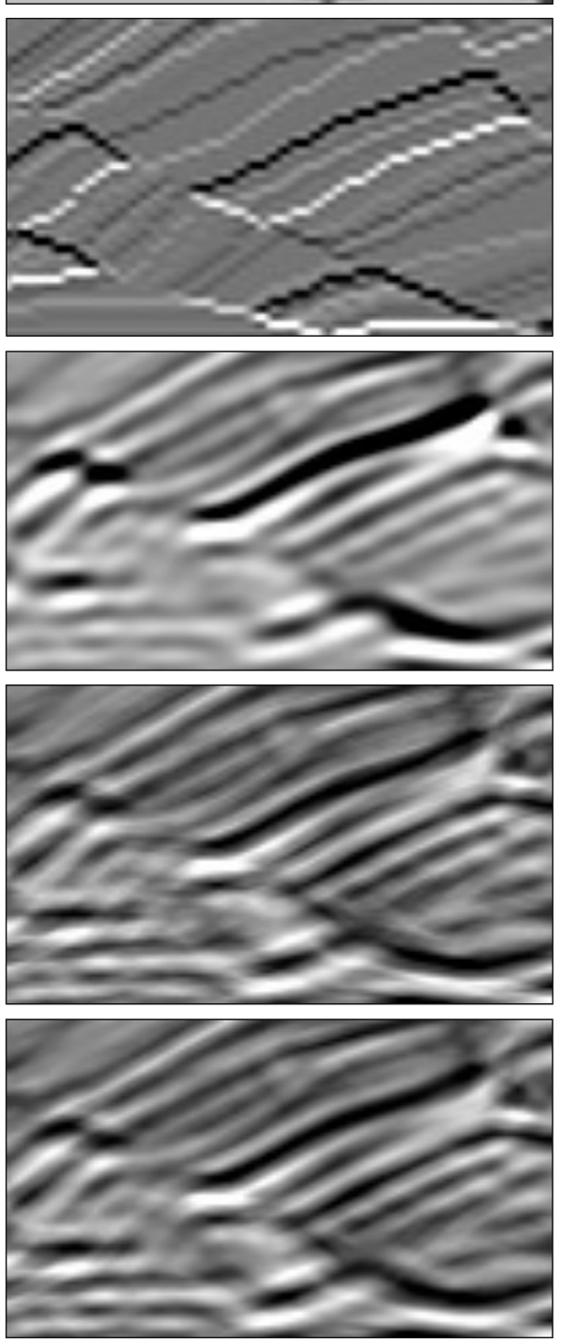
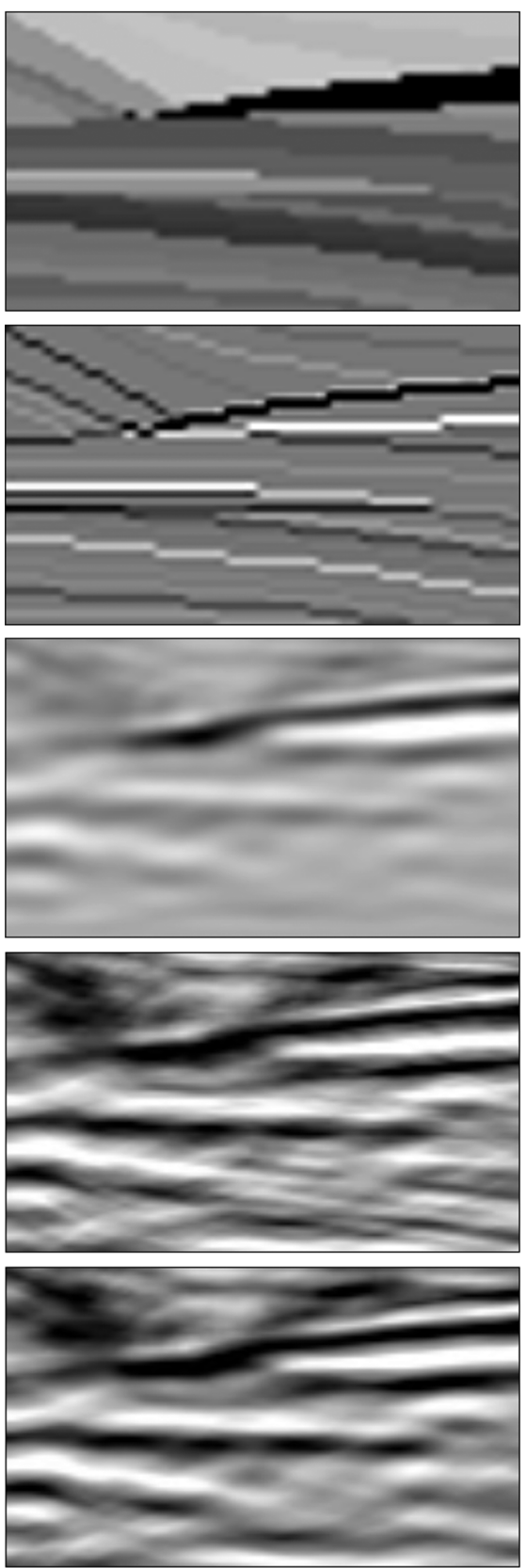

Figure 6. Magnifications of selected areas of the model and images, indicated by rectangles L (left column), M (middle column), and R (right column) in the previous figures. (a) True Marmousi model in Figure 1a, (b) reflectivity model, (c) reference CC image (with illumination compensation) in Figure 2b, (d) amplitude-weighted PC image in Figure 4a, and (e) the envelope-weighted PC image in Figure 4b. 
in the lower corners of the image (black arrows) and brings out the finely layered dipping structures below the salt intrusion in rectangle L (superimposed on a horizontal artifact that is visible in all images).

\section{Sensitivity parameter}

The actual value of parameter $\nu$ is not very critical to the migrated result. We observe that increasing the value of $\nu$ promotes a slightly enhanced sharpness in the images resulting from equations 11 and 12. For comparison, we show in Figure 7 the amplitude-weighted PC RTM image obtained with $\nu=1$ in equation 12 (compare with Figure 4a). Because the resolution is slightly better in Figure $4 a$, we chose $\nu=100$ in our other numerical tests.

\section{APPLICATION TO A FIELD DATA SET}

As a next step, we tested the proposed PC-based imaging conditions on a field data set from a seismic line of a 2D land survey in the Solimões Basin (Amazon, Brazil) acquired in 1984 as part of an exploratory program. Survey parameters were $50 \mathrm{~m}$ source and receiver sampling, 241 sources, 96 channels in split-spread configuration, $3 \mathrm{~s}$ recording time, $4 \mathrm{~ms}$ sampling interval, and $8-90 \mathrm{~Hz}$ field bandpass. The seismic data have a low-fold stack (maximum equal to 48). In the surveyed area, there are two major problems for seismic processing. The first one is caused by a low-velocity zone, which makes it difficult to perform adequate static corrections. The second problem is caused by a relatively shallow basalt sill, which makes it difficult to obtain a good seismic image beneath it. As a consequence of both problems, a low signalto-noise ratio is attained for the seismic events below the basalt sill. The amplitude equalization achieved by RTM with PC-based imaging conditions makes it a natural candidate in the attempt to enhance the seismic events in the deep portions of the image, below the basalt sill.

Before applying RTM, the seismic data are processed according to the following flow: static correction, geometric spreading correction, amplitude correction to equalize the lateral spatial variation of the traces, spiking deconvolution, spectral balancing, residual static correction (trying to better correct relatively high-frequency discontinuities), mute (on surface head waves), Radon transform (to interpolate missing traces), and band-pass filtering $(10-60 \mathrm{~Hz})$.

The RTM depth velocity model was built from a time migration velocity model through time-todepth conversion using vertical rays. The model is represented on a uniform mesh with $6.25 \mathrm{~m}$ sampling interval. The source pulse is a Ricker wavelet with a peak frequency of $20 \mathrm{~Hz}$, which reasonably approximates the true wavelet observed in the data.

Figure $8 \mathrm{a}-8 \mathrm{c}$ shows the illumination-compensated CC reference image (condition $I_{\mathrm{CC}}^{i c}(\mathbf{x})$ in equation 7), the amplitude-weighted PC image

c) (condition $I_{\mathrm{PC}}^{p}(\mathbf{x})$ in equation 11), and the envelope-weighted PC image (condition $I_{\mathrm{PC}}^{A}(\mathbf{x})$ in equation 12), respectively. As before, the PC images were obtained with $\nu=100$. As mentioned above,

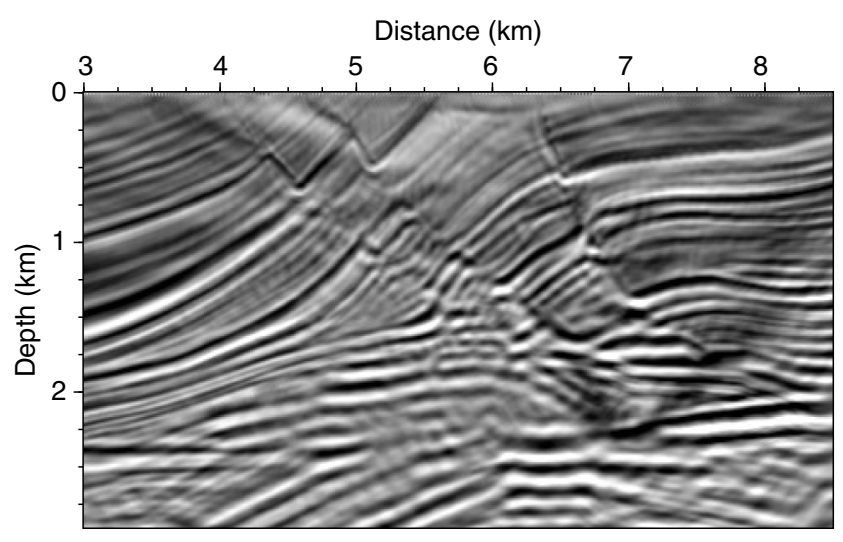

Figure 7. Amplitude-weighted PC RTM image (condition $I_{\mathrm{PC}}^{p}(\mathbf{x})$ in equation 11 ) with $\nu=1$.

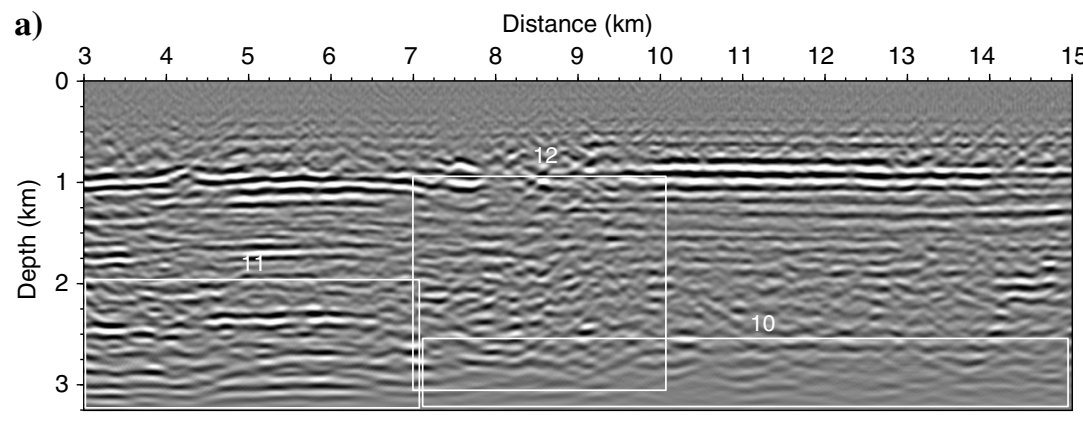

b) Distance $(\mathrm{km})$

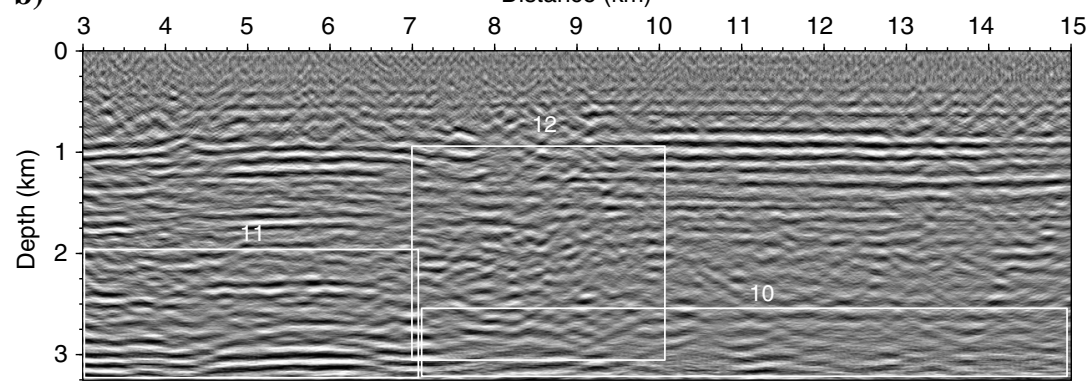

c) Distance $(\mathrm{km})$

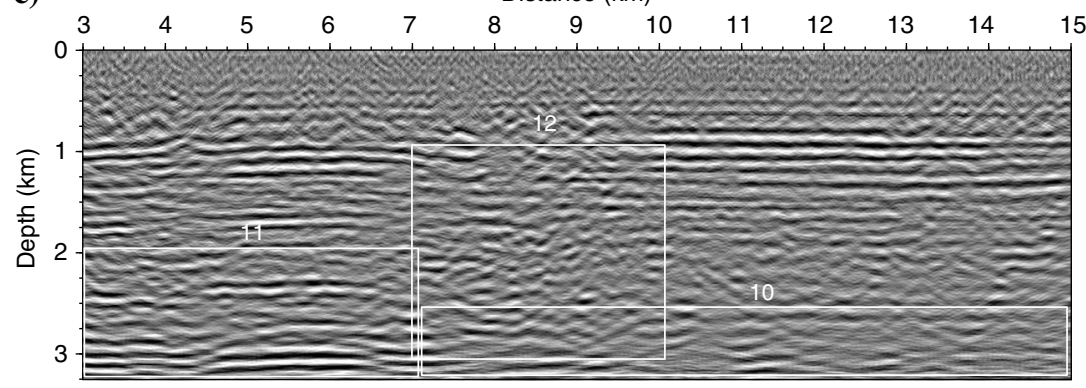

Figure 8. Field data: (a) classic crosscorrelation RTM image compensated for illumination (condition $I_{\mathrm{CC}}^{i c}(\mathbf{x})$ in equation 7). (b) Amplitude-weighted PC RTM image (condition $I_{\mathrm{PC}}^{p}(\mathbf{x})$ in equation 11) with $\nu=100$. (c) Envelope-weighted PC RTM image (condition $I_{\mathrm{PC}}^{A}(\mathbf{x})$ in equation 12) with $\nu=100$. The rectangles indicate the magnified areas. 


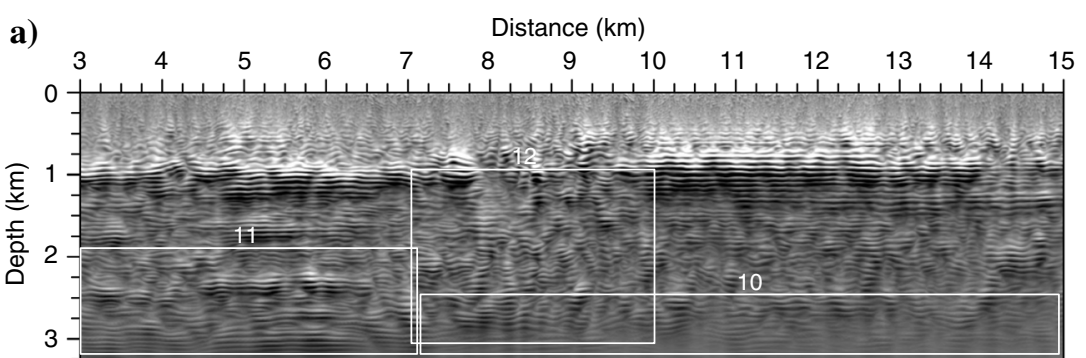

b)

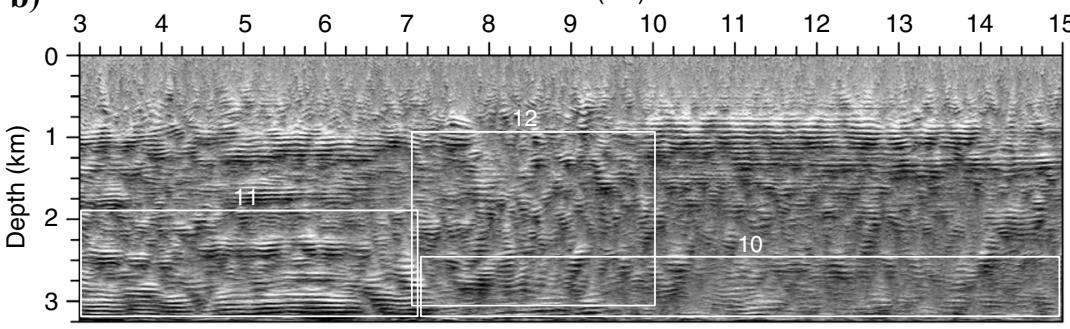

Figure 9. Field data: RTM images of Figure $8 \mathrm{a}$ and $8 \mathrm{~b}$ in amplitude-volume-processing display. (a) CC imaging condition with illumination compensation and (b) amplitudeweighted PC imaging condition. The rectangles indicate the magnified areas.

a)

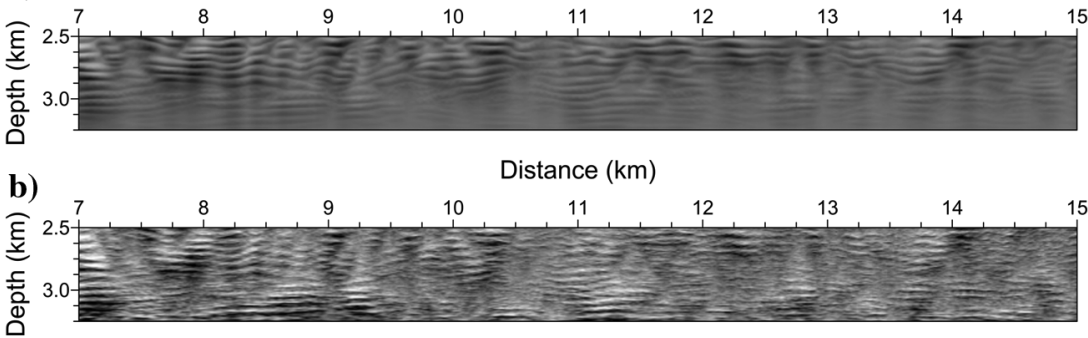

Figure 10. Field data: magnification of rectangle 10 in the RTM images of Figure 9 in amplitude-volume-processing display. (a) CC imaging condition with illumination compensation and (b) amplitude-weighted PC imaging condition.

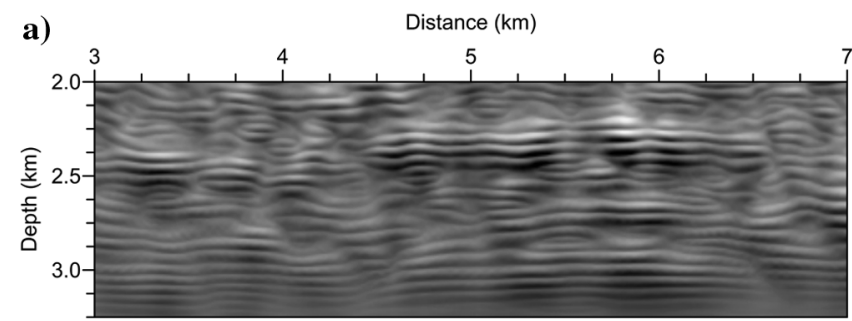

b)

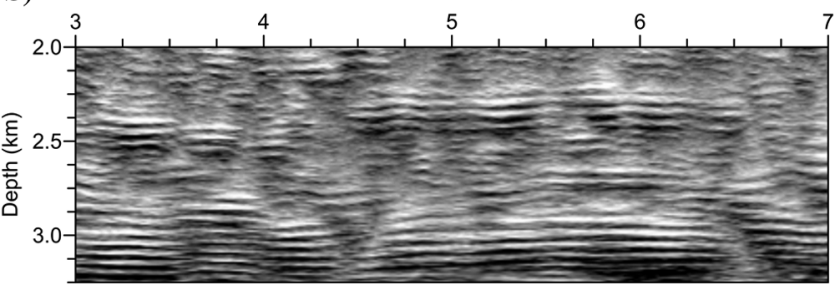

Figure 11. Field data: magnification of rectangle 11 in the RTM images of Figure 9 in amplitude-volume-processing display. (a) CC imaging condition with illumination compensation and (b) amplitudeweighted PC imaging condition. we refrain from removing low-frequency backscattering noise in all images to only visualize the effect of the imaging conditions.

We note that in this field-data example, the amplitude- and envelope-weighted PC images are very similar, even more so than in the synthetic-data example. All existing differences are extremely subtle and have no significance regarding the quality of the images. For this reason, we restrict the following discussion to a comparison between the $\mathrm{CC}$ and the amplitude-weighted PC images. To better visualize the differences between these two images, we present them in Figure 9 using a different visualization method, which uses an amplitude-volume processing technique adapted from Bulhões (1999). See also Bulhões and Amorim (2005) and Vernengo and Trinchero (2015). Our version consists of $a-\pi / 2$ phase rotation applied in the frequency domain to the modulus of each trace.

The CC and PC images show strong-amplitude reflection events at approximately $1 \mathrm{~km}$ depth, representing a shallow basalt sill. Below the sill, there are several differences between the two images. We have selected three areas where these differences are most prominent. They are highlighted by rectangles labeled 10, 11, and 12 in Figures 8 and 9, and Figures 10, 11, and 12 show the respective magnifications.

The most visible of these differences occurs at less than $2.5 \mathrm{~km}$ depth (see rectangle labeled 10 in Figures 8 and 9, magnified in Figure 10), where the PC image (Figure 9b) shows significantly enhanced amplitudes over the CC image (Figure 9a). In this way, the interpretation of reflectors at approximately $3 \mathrm{~km}$ depth in the distance interval $7-15 \mathrm{~km}$ becomes possible. In this deeper portion of the sections, the stratigraphic and structural features are hardly recognizable in the CC image (Figure 10a), but they are much better interpretable in the PC image (Figure 10b).

Another prominent example is constituted by the subvertical faults at approximately $3 \mathrm{~km}$ depth and between horizontal positions 3 and $7 \mathrm{~km}$ (rectangle 11, magnified in Figure 11). A possible interpretation of this portion of the section as composing a kind of "pull-up" geometry, limited by reverse faults, is much more easily justified in the PC image (Figure 11b) than in the CC image (Figure 11a). It is to be stressed that the existence of reverse faulting is in accordance with the known geology of the area (Barata and Caputo, 2007; Ribeiro and Lima, 2007).

Even above $2.5 \mathrm{~km}$ depth, where the $\mathrm{CC}$ and $\mathrm{PC}$ images have similar quality, they show different aspects of the same structures. For example, in the heavily faulted region between positions 7 and $10 \mathrm{~km}$ (rectangle 12, magnified in Figure 12), certainly an interpreter would be more confident to locate the fault planes and infer the relative offsets with the additional information of the PC image (Figure 12b) at hand than using just the CC image (Figure 12a) because several events are more easy to follow in the PC image. 


\section{DISCUSSION}

The PC imaging conditions presented in this work rely on the availability of the analytical signals of the source and receiver wavefields. Although the numerical propagation of the analytical signals is slightly more expensive than of their real counterparts, their knowledge is desirable for other purposes as well. For example, they can be used to decompose the wavefields into up- and downgoing constituents, which allows for the correlation of selected components to reduce backscattering (Shen and Albertin, 2015). An efficient procedure for the analytical-wavefield propagation has been proposed by Pestana and Revelo (2017). In this work, we have refrained from applying wavefield separation because we wanted to compare the PC imaging conditions with (real) crosscorrelation, which does not allow for this kind of decomposition.

Our main result is that the PC-based imaging conditions can help to improve the resolution and visibility of weak reflections, particularly in deeper regions of the model. This is in agreement with the findings of Schimmel (1999), who observes that PC shows higher sensitivity to coherence than CC regardless of the event amplitude, and of Barnes (2007), who notes that instantaneous phase as a seismic attribute produces finer layering details and improved reflection continuity. In fact, Barnes (2007) points out that the instantaneous

a)

a)

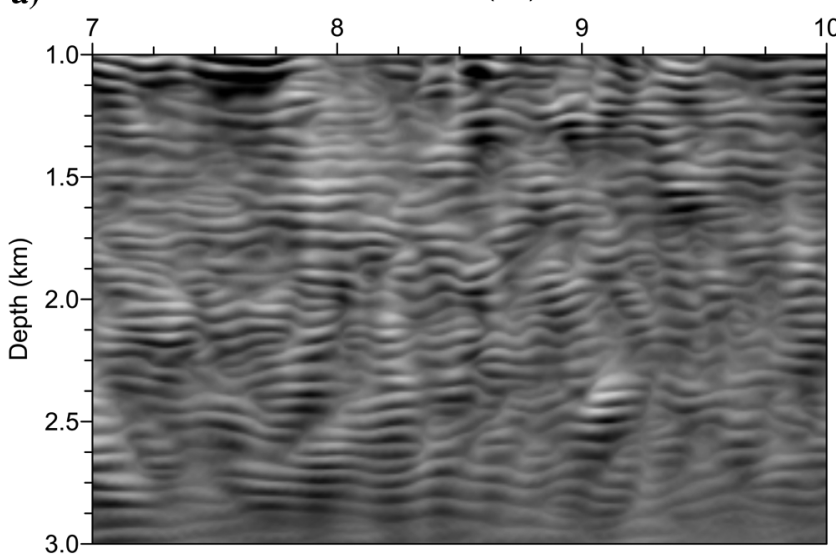

b)

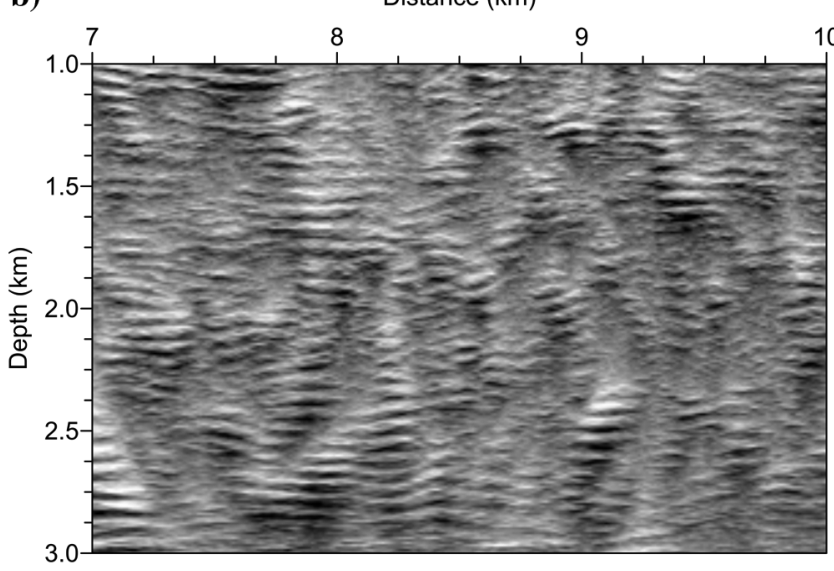

Figure 12. Field data: magnification of rectangle 12 in the RTM images of Figure 9 in amplitude-volume-processing display. (a) CC imaging condition with illumination compensation and (b) amplitudeweighted PC imaging condition. phase carries no amplitude information at all and, in this sense, acts like a kind of perfect automatic gain control, completely equalizing the amplitudes.

For this reason, PC-based imaging conditions can help to enhance weak-amplitude but phase-coherent events in the seismic image. This explains why in our examples, its most visible effect occurred in deeper regions, where geometric-spreading effects are strongest. Accordingly, in our real-data example, the main impact of the PC-based imaging conditions could be observed in the subbasalt portion of the image. We strongly believe that the biggest asset of the new imaging conditions is improved reflector visibility in poorly illuminated parts of the subsurface, particularly in problematic zones such as subsalt or subbasalt, where imaging remains a challenge.

\section{CONCLUSION}

$\mathrm{CC}$ and PC are independent approaches that provide complementary focusing criteria for RTM. Both PC and full-waveeld CC show maxima whenever there is waveform similarity, that is, whenever the migrated wavefields coincide in space and time at a scattering point in the subsurface. However, the phase coherence is sensitive just to waveform changes (and insensitive to waveform amplitude). Because of these properties, its inclusion into RTM can provide interesting additional information in the migrated images. Our numerical experiments on synthetic and field data indicate that the use of PC-based imaging conditions can lead to a refinement of the detected reflectors, allowing for increased resolution and reflector visibility, particularly in the deeper portions of the seismic images. In addition, the resolution gain can also help to enhance stratigraphic and structural features. In this way, the new imaging conditions can be instrumental to seismic interpretation.

The purely PC-based imaging condition is sensitive only to instantaneous phase changes and does not depend on the reflection amplitudes. This may lead to an enhancement of migration artifacts. Amplitude-weighted variations of this imaging condition preserve the desired properties of improving resolution while avoiding the enhancement of migration artifacts.

The PC-based imaging conditions can be computed simultaneously with conventional imaging conditions, once the analytical signals of the source and receiver wavefields have been propagated. Although this increases the cost of RTM by, at most, a factor of two, the additional cost is justified by the additional information obtained by the computation of several images with different imaging conditions in a single run. Moreover, the analytical wavefield can be used to decompose the involved wavefields into their up- and downgoing components, allowing for the simultaneous calculation of further additional imaging conditions, e.g., to reduce backscattering noise.

\section{ACKNOWLEDGMENTS}

We are grateful to associate editor A. Malcolm and four unknown reviewers for numerous valuable comments that helped to improve this manuscript. We thank $\mathrm{CNPq}$ for the financial support through the Instituto Nacional de Ciências e Tecnologia em Geofísica do Petróleo (INCT-GP). J. C. Costa, W. E. Medeiros, and J. Schleicher also thank $\mathrm{CNPq}$ for the research fellowships and associated grants. M. Schimmel thanks the Science without Borders Program for his PVE grant and Misterios project (CGL2013-48601-C2-1-R). Field 
data were provided by the Brazilian National Agency of Petroleum, Natural Gas and Biofuels (ANP) under Protocol No. 095221 (10 October 2016). Before applying RTM, the field data were processed using the UFRN academic license of PROMAX (Landmark Graphics Corporation).

\section{REFERENCES}

Barata, C. F., and M. V. Caputo, 2007, Geologia do petróleo da Bacia do Solimões. O "estado da arte": Proceedings of the 4th PDPETRO, ABPG, 1.1.0147-1-1.1.0147-10, http://www.portalabpg.org.br/PDPetro/ 4/resumos/4PDPETRO_1_1_0147-1.pdf, accessed 3 May 2018.

Barnes, A. E., 2007, A tutorial on complex seismic trace analysis: Geophysics, 72, no. 6, W33-W43, doi: 10.1190/1.2785048.

Bonatto, L., M. Schimmel, J. Gallart, and J. Morales, 2015, The uppermantle transition zone beneath the Ibero-Maghrebian region as seen by teleseismic PDS phases: Tectonophysics, 663, 212-224, doi: 10.1016/j. tecto.2015.02.002.

Bracewell, R. N., 1965, The Fourier transform and its applications: McGraw-Hill.

Bulhões, E. M., 1999, Técnica "Volume de Amplitudes" para mapeamento de feições estruturais: 6th International Congress of the Brazilian Geophysical Society, SBGf, no. 296, Expanded abstract, 1-4.

Bulhões, E. M., and W. N. Amorim, 2005, Princípio da sismocamada elementar e sua aplicação à técnica de volume de amplitudes (TecVA): 9th International Congress of the Brazilian Geophysical Society \& EXPOGEF, SBGf, 1382-1387, doi: 10.1190/sbgf2005-275.

Chattopadhyay, S., and G. A. McMechan, 2008, Imaging conditions for prestack reversetime migration: Geophysics, 73, no. 3, S81-S89, doi: 10.1190/1.2903822.

Claerbout, J. F., 1971, Toward a unified theory of reflector mapping: Geophysics, 36, 467-481, doi: 10.1190/1.1440185.

Costa, J. C., F. A. Silva Neto, M. R. M. Alcantara, J. Schleicher, and A. Novais, 2009, Obliquity-correction imaging condition for reverse time migration: Geophysics, 74, no. 3, S57-S66, doi: 10.1190/1.3110589.

D'Hour, V., M. Schimmel, A. F. Do Nascimento, J. M. Ferreira, and H. C. Lima Neto, 2015, Detection of subtle hydromechanical medium changes caused by a small-magnitude earthquake swarm in NE Brazil: Pure and Applied Geophysics, 173, 1097-1113, doi: 10.1007/s00024-015-1156-0.

Du, Q., C. Guo, Q. Zhao, X. Gong, C. Wang, and X.-Y. Li, 2017, Vectorbased elastic reverse time migration based on scalar imaging condition: Geophysics, 82, no. 2, S111-S127, doi: 10.1190/geo2016-0146.1.

Etgen, J., S. H. Gray, and Y. Zhang, 2009, An overview of depth imaging in exploration geophysics: Geophysics, 74, no. 6, WCA5-WCA17, doi: 10 $.1190 / 1.3223188$.

Gaudot, I., E. Beucler, A. Mocquet, M. Schimmel, and M. Le Feuvre, 2015, Statistical redundancy of instantaneous phases: Theory and application to the seismic ambient wavefield: Geophysical Journal International, 204, 1159-1163, doi: 10.1093/gji/ggv501.

Guitton, A., A. Valenciano, D. Bevc, and J. F. Claerbout, 2007, Smoothing image condition for shot-profile migration: Geophysics, 72, no. 3, S149S154, doi: 10.1190/1.2712113.

Haned, A., E. Stutzmann, M. Schimmel, S. Kiselev, A. Davaille, and A. Yelles-Chaouche, 2015, Global tomography using seismic hum: Geophysical Journal International, 204, 1222-1236, doi: 10.1093/gji/ggv516.
Holberg, O., 1987, Computational aspects of the choice of operator and sampling interval for numerical differentiation in large-scale simulation of wave phenomena: Geophysical Prospecting, 35, 629-655, doi: 10 .1111/j.1365-2478.1987.tb00841.x.

Moradpouri, F., A. Moradzadeh, R. Pestana, R. Ghaedrahmati, and M. S. Monfared, 2017, An improvement in wavefield extrapolation and imaging condition to suppress reverse time migration artifacts: Geophysics, 82, no. 6, S403-S409, doi: 10.1190/geo2016-0475.1.

Pestana, R. P., and D. Revelo, 2017, An improved method to calculate the analyticalwavefield for causal imaging condition: 87th Annual International Meeting, SEG, Expanded Abstracts, 4640-4644, doi: 10.1190/ segam2017-17664808.1.

Ribeiro, R. B., and T. C. S. Lima, 2007, Evidência da tectônica do Juruá em linhas 2D reprocessadas da Bacia do Solimões: Proceedings of the 4th PDPETRO, ABPG, 1.2.0209-1-1.2.0209-6, http://www.portalabpg.org. br/PDPetro/4/resumos/4PDPETRO_1_2_0209-1.pdf, accessed 3 May 2018.

Sava, P., 2007, Stereographic imaging condition for wave-equation migration: Geophysics, 72, no. 6, A87-A91, doi: 10.1190/1.2781582.

Schimmel, M., 1999, Phase cross-correlations: Design, comparisons, and applications: Bulletin of the Seismological Society of America, 89, 1366-1378.

Schimmel, M., and H. Paulssen, 1997, Noise reduction and detection of weak, coherent signals through phase-weighted stacks: Geophysical Journal International, 130, 497-505, doi: 10.1111/j.1365-246X.1997.tb05664.x.

Schimmel, M., E. Stutzmann, and J. Gallart, 2011, Using instantaneous phase coherence for signal extraction from ambient noise data a local to a global scale: Geophysical Journal International, 184, 494-506, doi: 10.1111/j.1365-246X.2010.04861.x.

Schleicher, J., J. C. Costa, and A. Novais, 2008, A comparison of imaging conditions for wave-equation shot-profile migration: Geophysics, 73, no. 6, S219-S227, doi: 10.1190/1.2976776.

Shen, P., and U. Albertin, 2015, Up-down separation using Hilbert transformed source for causal imaging condition: 85th Annual International Meeting, SEG, Expanded Abstracts, 4175-4179, doi: 10.1190/ segam2015-5862960.1.

Tanaka, S., 2005, Characteristics of PKP-Cdiff coda revealed by small-aperture seismic arrays: Implications for the study of the inner core boundary: Physics of the Earth and Planetary Interiors, 153, 49-60, doi: 10.1016/j. pepi.2005.05.007.

Vernengo, L., and E. Trinchero, 2015, Application of amplitude volume technique attributes, their variations, and impact: The Leading Edge, 34, 1246-1253, doi: 10.1190/tle34101246.1.

Wang, W., G. A. McMechan, C. Tang, and F. Xie, 2016, Up/down and P/S decompositions of elastic wavefields using complex seismic traces with applications to calculating Poynting vectors and angle-domain commonimage gathers from reverse time migrations: Geophysics, 81, no. 4, S181S194, doi: 10.1190/geo2015-0456.1.

Yan, J., and T. A. Dickens, 2016, Reverse time migration angle gathers using Poynting vectors: Geophysics, 81, no. 6, S511-S522, doi: 10.1190/ geo2015-0703.1.

Yoon, K., and K. Marfurt, 2006, Reverse-time migration using the Poynting vector: Exploration Geophysics, 37, 102-107, doi: 10.1071/EG06102.

Zhang, Y., and J. Sun, 2009, Practical issues in reverse time migration: True amplitude gathers, noise removal and harmonic source encoding: First Break, 26, 53-59, doi: 10.3997/1365-2397.2009002.

Zhang, Y., J. Sun, and S. Gray, 2007, Reverse-time migration: Amplitude and implementation issues: 77th Annual International Meeting, SEG, Expanded Abstracts, 2145-2149, doi: 10.1190/1.2792912. 